\title{
Caveolin-1 Is Critical for Lymphocyte Trafficking into Central Nervous System during Experimental Autoimmune Encephalomyelitis
}

\author{
Hao Wu, ${ }^{1}$ Ruixia Deng, ${ }^{1}$ Xingmiao Chen, ${ }^{1}$ Waiman Connie Wong, ${ }^{2}$ Hansen Chen, ${ }^{1}$ Lei Gao, ${ }^{1}$ Yichu Nie, ${ }^{3}$ \\ Wutian $\mathrm{Wu},{ }^{2,4,5}$ and Jiangang Shen ${ }^{1}$ \\ ${ }^{1}$ School of Chinese Medicine, University of Hong Kong, Hong Kong SAR, China, ${ }^{2}$ School of Biomedical Science, University of Hong Kong, Hong Kong SAR, \\ China, ${ }^{3}$ Guangzhou Quality R\&D Center of Traditional Chinese Medicine, School of Life Science, Sun Yat-sen University, Guangzhou, 510275, China, ${ }^{4}$ State \\ Key Laboratory of Brain and Cognitive Sciences, University of Hong Kong, Hong Kong SAR, China, and ${ }^{5}$ Joint Laboratory for CNS Regeneration, Jinan \\ University and University of Hong Kong, GHM Institute of CNS Regeneration, Jinan University, Guangzhou, 510632, China
}

Multiple sclerosis (MS) is a progressive autoimmune disease of the CNS with its underlying mechanisms not fully understood. In the present study, we tested the hypothesis that caveolin-1, a major membrane scaffolding protein, plays a critical role in the pathogenesis of experimental autoimmune encephalomyelitis, a laboratory murine model of MS. We found increased expression of caveolin-1 in serum and spinal cord tissues in association with disease incidence and severity in wild-type mice with active encephalomyelitis. After immunization, Cav-1 knock-out mice showed remarkable disease resistance with decreased incidence and clinical symptoms. Furthermore, Cav-1 knock-out mice had alleviated encephalitogenic T cells trafficking into the CNS with decreased expressions of adhesion molecules ICAM-1 and VCAM-1 within the lesions. In agreement with in vivo studies, in vitro knockdown of caveolin-1 compromised the upregulation of ICAM-1 in endothelial cells, leading to the amelioration of the transendothelial migration of pathogenic $\mathrm{T}_{\mathrm{H}} 1$ and $\mathrm{T}_{\mathrm{H}} 17$ cells. Together, those results indicate that caveolin-1 serves as an active modulator of CNS-directed lymphocyte trafficking and could be a therapeutic target for neuroinflammatory diseases, such as multiple sclerosis.

Key words: caveolin-1; experimental autoimmune encephalomyelitis; ICAM-1; lymphocyte trafficking; VCAM-1

Significance Statement

The hallmark feature of neuroinflammatory diseases is the massive infiltrations of encephalitogenic leukocytes into the CNS parenchyma, a process that remains largely unclear. Our study demonstrates the critical contribution of caveolin-1 to encephalomyelitis pathogenesis and CNS-directed lymphocyte trafficking by modulation of adhesion molecules ICAM-1 and VCAM-1, highlighting the pathological involvement of caveolin-1 in neuroinflammatory diseases.

\section{Introduction}

Multiple sclerosis (MS) is a progressive autoimmune disease of the CNS system with global significance. The disease is characterized by perivascular leukocyte extravasation into CNS paren-

Received Oct. 12, 2015; revised April 1, 2016; accepted April 7, 2016.

Author contributions: J.S. and W.W. designed research;H.W. and R.D. performed research; R.D., X.C., W.C.W., and Y.N. contributed unpublished reagents/analytic tools; H.W., R.D., X.C., W.C.W., H.C., L.G., Y.N., W.W., and J.S. analyzed data; H.W. and J.S. wrote the paper.

This work was supported by Hong Kong Research Council Grant RGC GRF 17118511 to J.S. and University of Hong Kong Seed Fund for Basic Research 201311159015 to J.S. We thank Professor Scott W. Burchiel (University of New Mexico) for proofreading the manuscript; Professor Shuguang Wu (Southern Medical University) for valuable suggestions; and the Medical Faculty Core Facility at the University of Hong Kong for technical support.

The authors declare no competing interests.

Correspondence should be addressed to either Prof. Jiangang Shen or Prof. Wutian Wu, Li Ka Shing Faculty of Medicine, University of Hong Kong, Hong Kong SAR, China, E-mail: shenjg@hku.hk or wtwu@hku.hk.

DOI:10.1523/JNEUROSCI.3734-15.2016

Copyright $\odot 2016$ the authors $\quad 0270-6474 / 16 / 365193-07 \$ 15.00 / 0$ chyma, causing demyelination and axonal damage as well as neurological defects, including paresis or paralysis. Experimental autoimmune encephalomyelitis (EAE) is a widely adopted laboratory model that mimics key features of MS, including CNSdirected leukocyte infiltrations and demyelination (Goverman, 2009). During disease pathogenesis, immune cell trafficking occurs when encephalitogenic leukocytes cross the blood-brain barrier (BBB) into the CNS parenchyma (Engelhardt and Ransohoff, 2012; Kawakami et al., 2012). This process requires intensive interactions of primed leukocytes with BBB microvascular endothelial cells. For instance, proinflammatory stimuli activate the $\mathrm{BBB}$, causing microvascular endothelial cells to upregulate the expression of adhesion molecules that bind to receptors on leukocyte surfaces for leukocyte firm adhesion and diapedesis. Among various adhesion molecules, vascular cell adhesion molecule-1 (VCAM-1) and intracellular adhesion molecule-1 
A

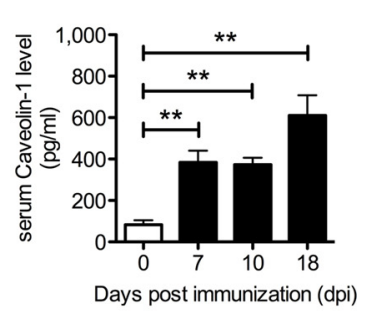

B

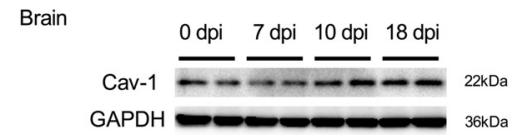

C

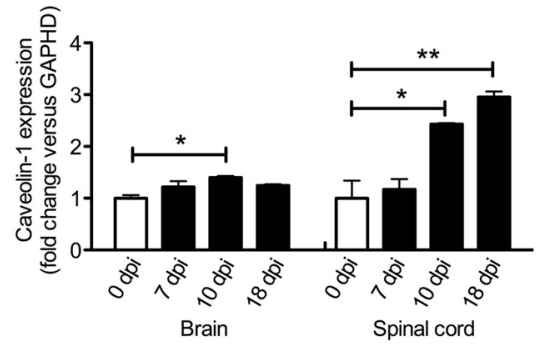

D

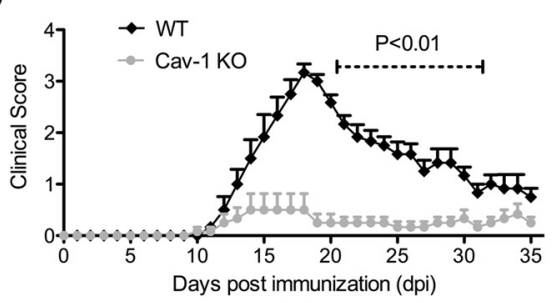

H

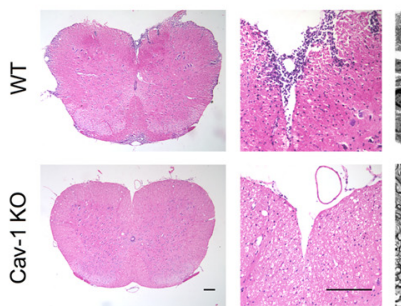

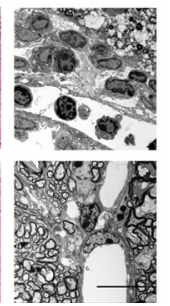

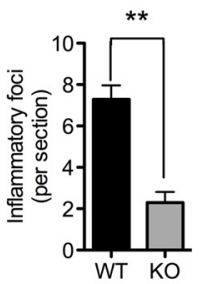

E

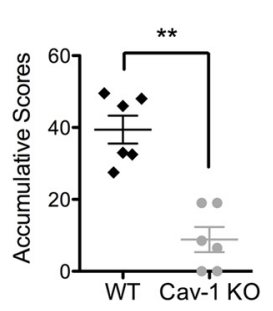

$\mathbf{F}$

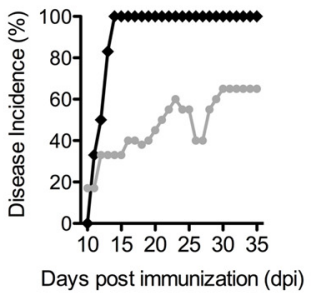

I

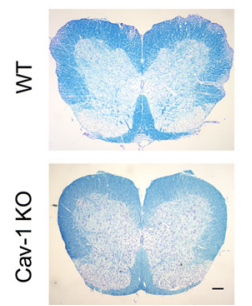

G

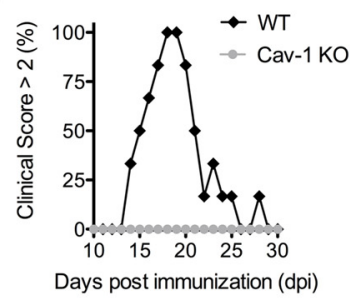

Figure 1. Cav-1-deficient mice are resistant to active EAE. $\boldsymbol{A}$, ELISA detection of serum Cav-1 level in WT mice after active immunization. $\boldsymbol{B}$, Western blot detection of the time-dependent response of Cav-1 expression in brain and spinal cord of WT mice after immunization. $\boldsymbol{C}$, Statistical analysis of $\boldsymbol{B}$. D, Clinical scores of immunized WT and Cav-1 K0 mice after immunization. $\boldsymbol{E}$, Cumulative scores of WT and Cav-1 KO mice for 35 dpi. $\boldsymbol{F}$, Active EAE incidence in both genotypes. $\boldsymbol{G}$, Incidence of severe EAE symptoms (defined as clinical score $\geq 2.5$ ) in both genotypes. $\boldsymbol{H}, \boldsymbol{I}$, Histological examination of inflammatory lesions and demyelination in the lumbar spinal cord of WT and Cav-1 KO mice by H\&E staining, Luxol fast blue staining (scale bar, $100 \mu \mathrm{m}$ ), and electronic microcopy (scale bar, $5 \mu \mathrm{m})$ at peak of disease (18 dpi). Data represent three $(\boldsymbol{A}-\boldsymbol{C}, \boldsymbol{H}, \boldsymbol{I})$ and four $(\boldsymbol{D}-\boldsymbol{G})$ independent experiments. Data are mean \pm SEM. ${ }^{*} p<0.05$, determined by one-way AN0VA $(\boldsymbol{A}-\boldsymbol{C})$ or unpaired Student's $t$ test $(\boldsymbol{D}-\boldsymbol{I}) .{ }^{* *} p<0.01$, determined by one-way ANOVA $(\boldsymbol{A}-\boldsymbol{C})$ or unpaired Student's $t$ test $(\boldsymbol{D}-\boldsymbol{I})$.

(ICAM-1) are two distinct ligands that account for the leukocyte transmigration. During inflammation, CNS vascular endothelial cells increase expression of ICAM-1 and VCAM-1, which can bind to lymphocyte function-associated antigen-1 (LFA-1) and very late antigen-1 (VLA-4) on leukocyte surfaces and guide efficient leukocyte infiltrations into CNS parenchyma (Greenwood et al., 2011). However, the underlying molecular mechanisms associated with leukocyte diapedesis and its impacts on CNS demyelination still remain unclear.

Caveolin-1 (Cav-1), a scaffolding protein of caveolae, has been reported to participate in regulating both vascular integrity (Tian et al., 2012) and intracellular trafficking (Ge and Pachter, 2004; Engel et al., 2011) actively. In previous studies, although the increased expression of Cav-1 in EAE and other neurodegenerative diseases has been reported, its functions remain largely unclear (Gaudreault et al., 2004; Shin et al., 2005; Kim et al., 2006; Nag et al., 2009). Herein, in the present study, we aim to explore the potential functions of Cav-1 in EAE pathogenesis and its underlying mechanisms. Our results demonstrate that Cav-1 participates in EAE development and facilitates lymphocyte trafficking into CNS by modulating adhesion molecules ICAM-1 and VCAM-1.

\section{Materials and Methods}

Mice. Cav-1 knock-out mice (KO) (Cav1 ${ }^{\mathrm{tm} 1 \mathrm{Mls}} / \mathrm{J}$; The Jackson Laboratory) were backcrossed with C57BL/6N mice to generate Cav-1 KO mice and wild-type (WT) littermates. All animal experiments were approved by the Committee on the Use of Live Animals in Teaching and Research at the University of Hong Kong.

Active EAE induction. Female WT or Cav-1 KO mice were subcutaneously immunized with $200 \mu \mathrm{g} \mathrm{MOG}_{35-55}$ in complete Freund's adjuvant ( $5 \mathrm{mg} / \mathrm{ml}$, Sigma-Aldrich). Pertussis toxin (200 ng, List Biological Laboratories) was injected intraperitoneally twice on day 0 and $48 \mathrm{~h}$ later. EAE symptoms were scored daily as follows: 0 , no clinical signs; 0.5 , partially limp tail; 1 , paralyzed tail; 1.5 , hindlimb paresis or loss in coordinated movement; 2 , loss in coordinated movement and hindlimb paresis; 2.5 , one hindlimb paralyzed; 3 , both hindlimbs paralyzed; 4 , hindlimbs paralyzed, weakness in forelimbs; 5 , forelimbs paralyzed.

ELISA. Serum were collected at indicated time points and detected for caveolin-1 according to manufacturer's instructions (Cusabio). Absorbance was measured at $450 \mathrm{~nm}$ with reference wavelength at $620 \mathrm{~nm}$ (Bio-Rad microplate reader).

Western blot analysis. CNS microvessels were isolated as previously described (Gu et al., 2012). Cell and tissue lysates were fractionated by $10 \%$ SDS-PAGE gel, electrotransfered onto PVDF membrane (Millipore), and immunoblotted with primary antibodies ICAM-1 (Santa Cruz Biotechnology), Cav-1 (Cell Signaling Technology), and GAPDH (Millipore) following HRP-conjugated secondary antibodies (Santa Cruz Biotechnology). The signals were visualized by advanced ECL detection kit (BD Biosciences) and analyzed by Gel-Doc (Bio-Rad).

Sample preparation for immunofluorescent staining. At the peak of disease (day 18 post immunization [ $18 \mathrm{dpi}]$ ), brains and spinal cords were isolated and fixed, followed by embedding in OCT, and cut into $30 \mu \mathrm{m}$ sections. Sections were stained with primary antibodies ICAM-1 (Santa 

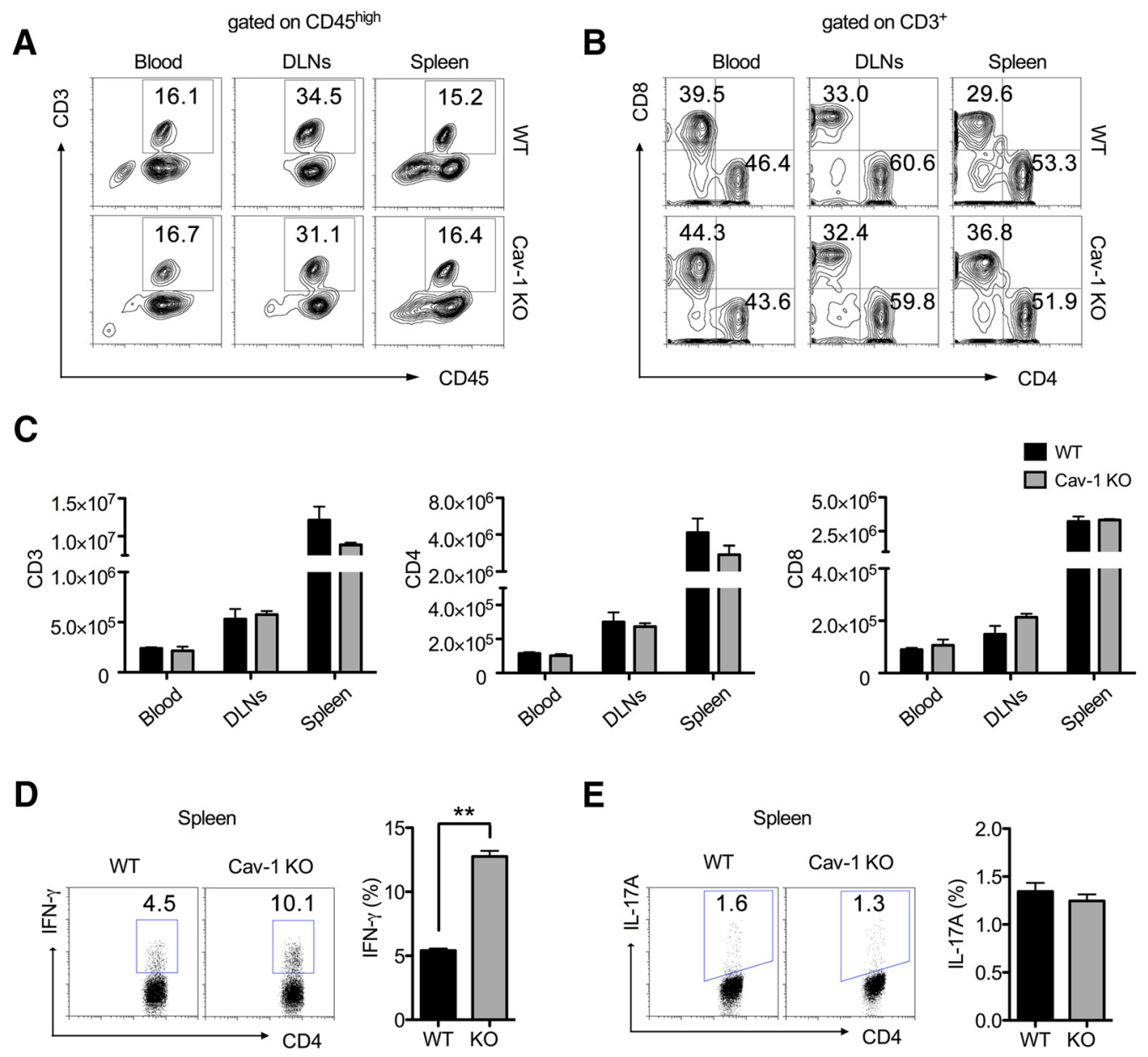

Figure 2. Cav-1 K0 does not suppress T-cell activation in vivo. A, B, Flow cytometry analysis of CD3, CD4, and CD8 T-cell population in peripheral blood, draining lymph nodes, and spleen of WT and Cav-1 K0 mice $10 \mathrm{dpi}$. C, Statistical analysis of the absolute numbers of $\boldsymbol{A}$ and $\boldsymbol{B}$. D, $\boldsymbol{E}$, Flow cytometry analysis of the percentage of IFN- $\gamma$ (left) or IL-17A (right) producing $\mathrm{T}_{H} 1$ or $\mathrm{T}_{H} 17$ cells in splenocytes (10 dpi). $\boldsymbol{A}-\boldsymbol{E}$, Data are from three independent experiments. Data are mean \pm SEM. ${ }^{* *} p<0.01$ (unpaired Student's $t$ test).

Cruz Biotechnology), VCAM-1 (Santa Cruz Biotechnology), GFAP (Sigma-Aldrich), Cav-1 (Cell Signaling Technology), and von Willebrand factor (Abcam). Immunofluorescent images were captured using a confocal laser scanning microscope LSM 780 (Carl Zeiss).

Sample preparation for immunohistochemistry and eletron microscopy. For histological and IHC staining, paraffin sections were cut into $8 \mu \mathrm{m}$ slices and stained with Luxol fast blue or H\&E. Demyelination (defined as the demyelinating area percentage in total white matter area) and inflammatory foci (defined as continuous clustered leukocytes at one site in the white matter) were quantified by ImageJ software. For EM processing, L4-L6 spinal cord blocks were embedded into Epon and cut into $90 \mathrm{~nm}$ ultrathin sections with 3\% uranyl acetate and 1\% lead citrate staining. Digital images were captured by electron microscopy (TEM, Phillips model 208).

Isolation of CNS infiltrating mononuclear cells. Mice were killed on 18 dpi with brain and spinal cord tissues dissected out and homogenized. Mononuclear single-cell suspensions were isolated from the interphase of 30\% and 70\% Percoll gradient (GE Healthcare Life Sciences).

Flow cytometry. Isolated single-cell suspensions were surface-stained with CD45-PE, CD3e-FITC, CD4-Pacific Blue, and CD8a-Alexa700 (eBioscience). For intracellular staining, cells were isolated from spleen (10 dpi), brain and spinal cord (20 dpi), restimulated with PMA and ionomycin in the presence of brefeldin A, and surface-stained with CD45-PE and CD4-Pacific blue. Cells were then fixed and permeabilized followed by staining with intracellular antibodies IFN $\gamma$-Alexa700a, IL17A-FITC, or isotype controls (BD Biosciences). Cell numbers were determined by BD LSR II using CountBright Beads (Invitrogen).
Ex vivo $T_{H} 1$ and $T_{H} 17$ cell polarization. On $10 \mathrm{dpi}, \mathrm{CD} 4^{+} \mathrm{T}$ cells from the draining lymph nodes of immunized WT mice were sorted (Miltenyi Biotec) and restimulated with $\mathrm{MOG}_{35-55}(20 \mu \mathrm{g} / \mathrm{ml})$ for $72 \mathrm{~h}$. For $\mathrm{T}_{\mathrm{H}} 1$ cell polarization, the following supplements were added: $10 \mu \mathrm{g} / \mathrm{ml}$ antiIL-4 and $10 \mathrm{ng} / \mathrm{ml} \mathrm{rmIL-12}$. For $\mathrm{T}_{\mathrm{H}} 17$ cells, the following supplements were added: $10 \mu \mathrm{g} / \mathrm{ml}$ anti-IL-4 and $10 \mu \mathrm{g} / \mathrm{ml}$ anti-IFN $\gamma, 20 \mathrm{ng} / \mathrm{ml}$ rmIL-6, $10 \mathrm{ng} / \mathrm{ml} \mathrm{rmIL-23,} 10 \mathrm{ng} / \mathrm{ml} \mathrm{rmIL-} \beta$, and $3 \mathrm{ng} / \mathrm{ml} \mathrm{rhTGF}-\beta 1$. Neutralizing antibodies and cytokines were obtained from Miltenyi Biotec.

Cav-1 knockdown and transendothelial electrical resistance (TEER) measurement. Mouse microvascular endothelial cells bEnd3 (ATCC) were seeded onto 6-well culture inserts $(8.0 \mu \mathrm{m}$, Millipore). After the cells were cultured for $24 \mathrm{~h}$, siRNA was used to knock down Cav-1, and a scramble siRNA was used as control. Cell membrane resistance (TEER) was measured from day 1 to day 6 using an epithelial voltohmmeter EVOM2 (World Precision Instruments) following the manufacturer's instructions. The TEER value per unit $\left(1 \mathrm{~cm}^{2}\right)$ was normalized as follows: $\mathrm{R}_{\text {sample }}\left(\Omega \mathrm{cm}^{2}\right)=\left(\mathrm{R}_{\text {measure }}-\mathrm{R}_{\text {blank }}\right) \times$ Effective Membrane Area.

Transendothelial adhesion and migration assay. bEnd3 cells were seeded onto 12-well culture inserts (8.0 $\mu \mathrm{m}$, Millipore). After Cav-1 knockdown, cells were stimulated with hTNF- $\alpha(25 \mathrm{ng} / \mathrm{ml})$ and IL- $1 \beta$ (10 ng/ml) (Peprotech) for $24 \mathrm{~h}$. Polarized $\mathrm{T}_{\mathrm{H}} 1$ and $\mathrm{T}_{\mathrm{H}} 17$ cells were added to the upper chamber of culture insert, allowing coincubation for $24 \mathrm{~h}$. The transmigrated cells from the lower chamber were quantified by FACS with CountBright Beads (Invitrogen).

Statistical analysis. Statistical analysis was assessed using an unpaired Student's $t$ test for two group comparison or one-way ANOVA for mul- 

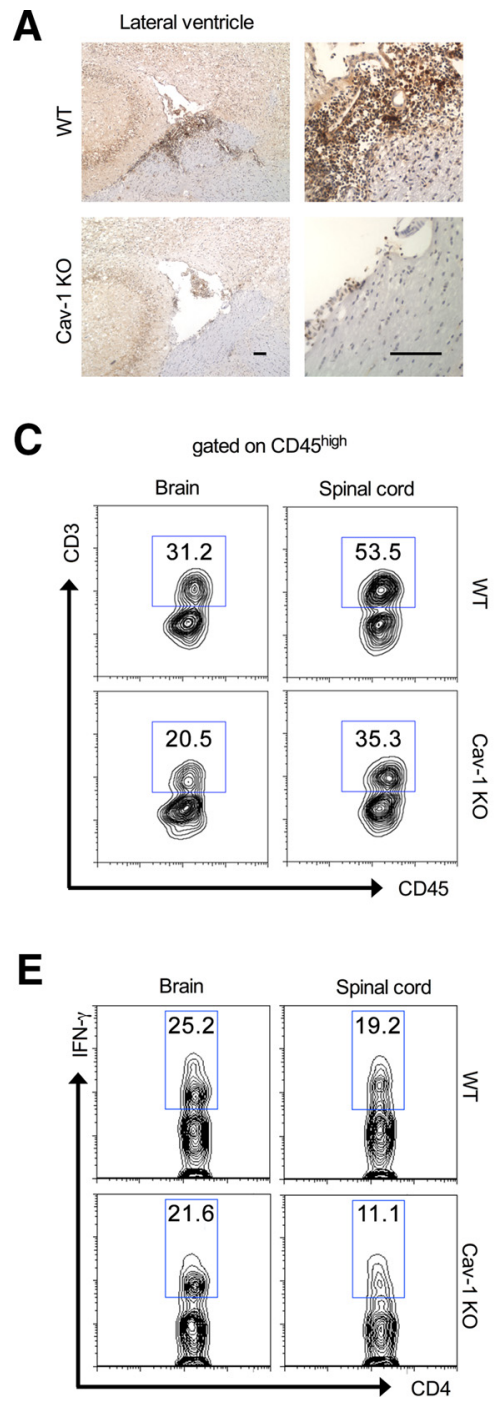

B
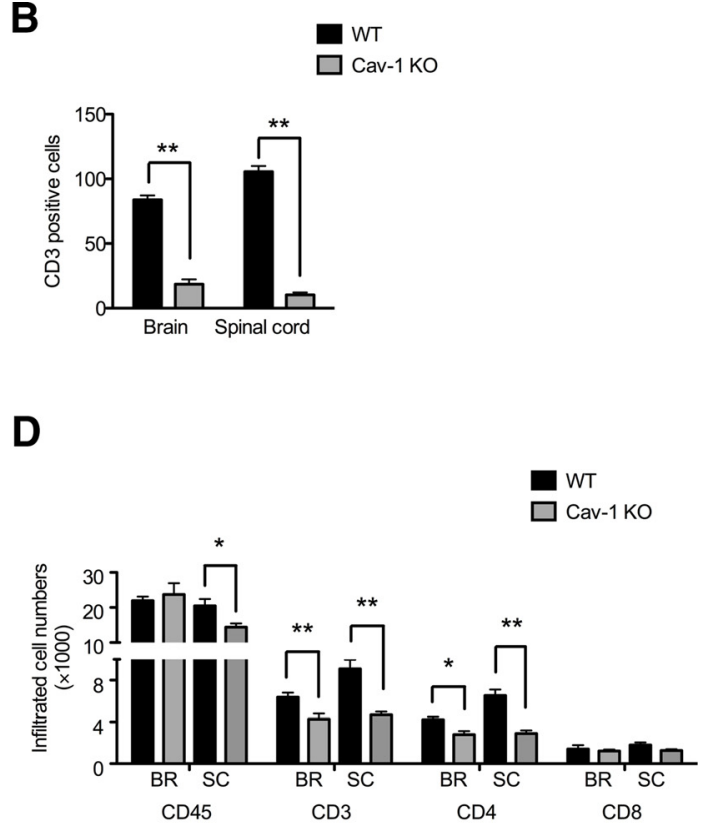

Figure 3. Cav-1 KO limits CNS-directed T lymphocyte extravasation. $\boldsymbol{A}$, Immunohistochemistry staining of CD3 ${ }^{+}$cells in cerebral parenchyma (near choroid plexus within lateral ventricle) and spinal cord of WT and Cav-1 K0 mice (18 dpi). B, Statistical analysis of $\boldsymbol{A}$. C, D, The infiltrated percentage and absolute numbers of CD3, CD4, and CD8 T cells in the brain and spinal cord of immunized WT and Cav-1 KO mice (18 dpi) by FACS.E, F, Flow cytometry analysis of the percentage and cell numbers of $\mathrm{T}_{H} 1$ and $\mathrm{T}_{H} 17$ cells in the CNS of both genotypes (18 dpi). Scale bar, $100 \mu \mathrm{m}$. Data represent five $(\boldsymbol{A}, \boldsymbol{B})$ and three $(\boldsymbol{C}-\boldsymbol{F})$ independent experiments. Data are mean \pm SEM. ${ }^{*} p<0.05$ (unpaired Student's $t$ test). ${ }^{* *} p<0.01$ (unpaired Student's $t$ test).

tiple group comparison. Data were presented as mean \pm SEM. For data analysis, $p<0.05$ was considered to be significant statistically.

\section{Results}

\section{Cav-1 deficient mice were resistant to active EAE}

We first investigated the dynamic changes of Cav- 1 in serum and CNS tissues in WT mice after immunization. We found that serum Cav-1 levels were coincidently increased with the disease progression (Fig. 1A). In agreement with serum Cav-1 secretion, the expression of Cav-1 in the spinal cord was also upregulated and reached its maximum at $18 \mathrm{dpi}$ (Fig. $1 B, C$ ).

We next investigated the roles of Cav-1 in EAE progression by using Cav-1 KO mice and its WT littermates. After immunization, Cav-1 KO mice had significantly alleviated clinical symptoms (Fig. 1D,E), which were accompanied with a reduced disease incidence (Fig. $1 F$ ). Most of the WT mice exhibited severe symptoms with paralyzed hindlimb (clinical score $>2$ ), while none of Cav-1 KO mice encountered this neurological defect throughout the disease course (Fig. 1G). We then examined inflammatory and demyelinating lesions, within the CNS in both genotypes at the peak time of disease progress (18 dpi). WT mice revealed characteristic demyelination in the white matter of spinal cord lesions with massive leukocyte adherence and extravasations as well. In contrast, Cav-1 KO mice had significantly reduced demyelination and inflammatory infiltrations. EM images further demonstrated the reduced lesions and wellpreserved myelin sheath structures in Cav-1 KO mice (Fig. $1 H, I)$. These results strongly suggest the significant requirement of Cav-1 in EAE pathogenesis.

\section{Cav-1 KO T lymphocytes responded to active \\ EAE immunization}

After immunization, antigen-specific $\mathrm{T}$ cells are primed in the periphery before migration into the CNS (Zamvil and Steinman, 1990). To investigate the effects of Cav-1 on early T-cell priming, we evaluated the encephalitogenic T-cell populations in the periphery at disease onset ( $10 \mathrm{dpi}$ ). FACS revealed that Cav-1 deficiency could maintain the cell population and percentage of $\mathrm{CD} 3^{+}, \mathrm{CD}^{+}{ }^{+}$, and $\mathrm{CD} 8{ }^{+} \mathrm{T}$ cells in peripheral blood, draining lymph nodes and spleen (Fig. $2 A-C$ ) at disease onset. We also measured the subpopulation of $\mathrm{T}_{\mathrm{H}} 1$ and $\mathrm{T}_{\mathrm{H}} 17 \mathrm{CD} 4^{+} \mathrm{T}$ cells in 
A
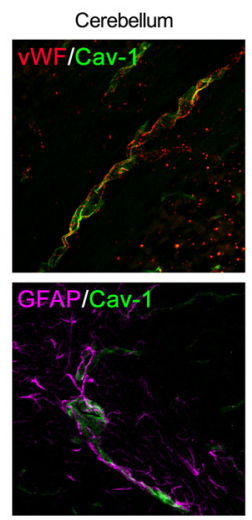

D

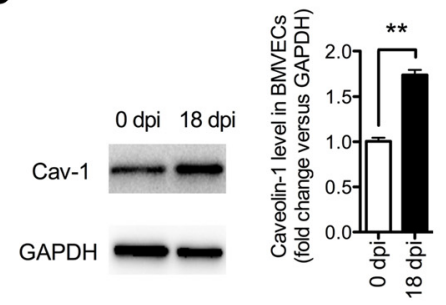

B
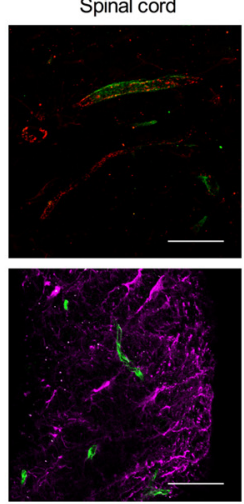

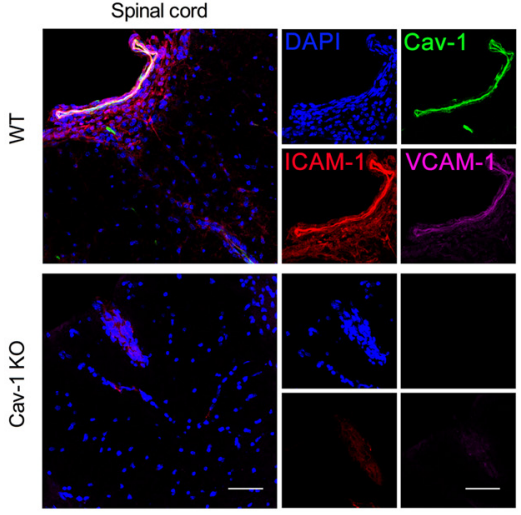

C

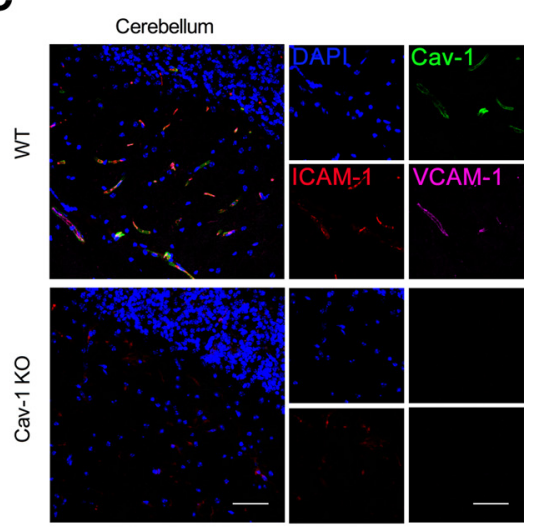

E

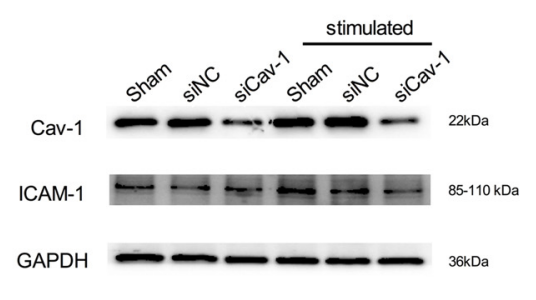

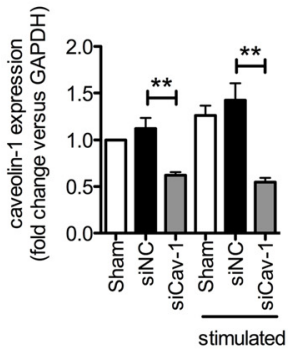

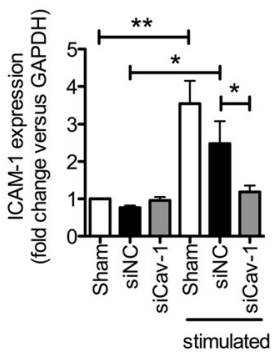

F

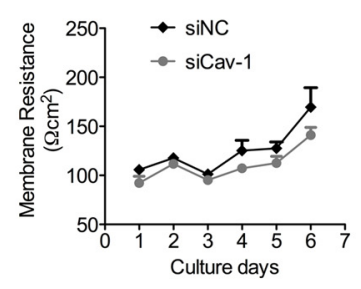

G

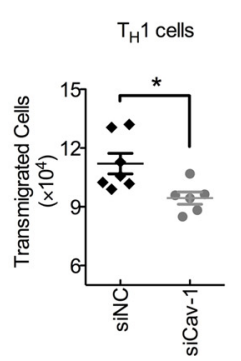

H

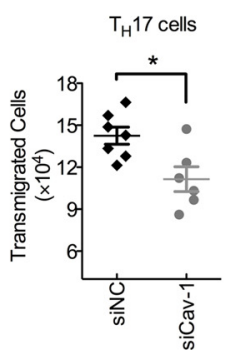

Figure 4. Endothelial Cav-1 is essential for lymphocyte transendothelial migration. $A$, Immunofluorescent costaining of Cav-1 with GFAP and vWF in the white matter of cerebellum and spinal cord of WT mice (18 dpi). B, C, Immunofluorescent costaining of Cav-1 with ICAM-1 and VCAM-1 in the white matter of spinal cord and cerebellum of immunized WT and Cav-1 K0 mice (18 dpi). $\boldsymbol{D}$, Western blot analysis of Cav-1 expression in CNS-derived microvessels of WT mice after immunization. $\boldsymbol{E}$, Western blot analysis of the expression of Cav-1 and ICAM-1 in bEnd3 cells. $\boldsymbol{F}$, TEER measurement of bEnd3 cells after Cav-1 knockdown. $\boldsymbol{G}, \boldsymbol{H}$, FACS analysis of the transmigrated $\mathrm{T}_{H} 1$ and $\mathrm{T}_{H} 17$ cell numbers with endothelial Cav- 1 knockdown. Scale bar, $50 \mu \mathrm{m}$. Data represent three $(\boldsymbol{A}-\boldsymbol{F})$ and two $(\boldsymbol{G}, \boldsymbol{H})$ independent experiments. Data are mean \pm SEM. ${ }^{*} p<0.05$, determined by unpaired Student's test $(\boldsymbol{D}, \boldsymbol{G}, \boldsymbol{H})$ or one-way ANOVA $(\boldsymbol{E}) .{ }^{* *} p<0.01$, determined by unpaired Student's $t$ test $(\boldsymbol{D}, \boldsymbol{G}, \boldsymbol{H})$ or one-way ANOVA $(\boldsymbol{E})$.

spleen (10 dpi). Cav-1 KO mice had a similar proportion of $\mathrm{T}_{\mathrm{H}} 17$ cells and an even higher percentage of $\mathrm{T}_{\mathrm{H}} 1$ cells in spleens compared with WT littermates (Fig. 2D,E). These results indicate that Cav-1 KO does not impair T-cell responses in the periphery at the early priming stage.

\section{Cav-1 deficiency impaired CNS-directed encephalitogenic T-cell extravasation}

After priming in the periphery, encephalitogenic leukocytes transmigrate across vascular endothelial cells into CNS parenchyma for a second wave of damage. Because Cav-1 did not impair the lymphocyte activation in the periphery, we next tested the effects of Cav-1 on CNS-directed lymphocyte trafficking. Compared with WT littermates, Cav-1 KO mice had a dramatic decrease in the number of infiltrated $\mathrm{CD}^{+}{ }^{+} \mathrm{T}$ cells in cerebral parenchyma and the white matter of spinal cord (Fig. $3 A, B$ ). FACS analysis further suggested that Cav-1 KO mice had a lower frequency of infiltrated $\mathrm{CD}^{+}{ }^{+} \mathrm{T}$ cells, predominantly $\mathrm{CD} 4{ }^{+} \mathrm{T}$ cells in the spinal cord compared with WT littermates (Fig. $3 C, D)$. Furthermore, Cav-1 KO mice had fewer infiltrations of
IFN- $\gamma$-producing $\mathrm{T}_{\mathrm{H}} 1$ cells and IL-17A-producing $\mathrm{T}_{\mathrm{H}} 17$ cells in the spinal cord (Fig. 3E,F). These results suggest that Cav-1 could be essential for the recruitment of pathological CD4 ${ }^{+} \mathrm{T}$ cells, specifically $\mathrm{T}_{\mathrm{H}} 1$ and $\mathrm{T}_{\mathrm{H}} 17$ cells, into the spinal cord. The diminished diapedesis of encephalitogenic $\mathrm{T}$ cells in the spinal cord of Cav-1 KO mice might contribute to its lower disease susceptibility.

\section{Endothelial Cav-1 was required for lymphocyte transmigration}

Upregulation of adhesion molecule expression on inflamed vascular endothelial cells is highly associated with the processes of lymphocyte extravasation into the CNS (Bullard et al., 2007; Steiner et al., 2010). To explore the functions of Cav-1 in this aspect, we examined the distribution of Cav-1 within CNS lesions. Immunofluorescent images revealed that Cav-1 was highly colocalized with $\mathrm{vWF}^{+}$vascular structures (Fig. $4 A$ ) but not with $\mathrm{GFAP}^{+}$astrocytes in the white matter of spinal cord and cerebellum, which implicates the potential role of Cav-1 in regulating vascular functions. We next examined the expression of adhesion 
molecule ICAM-1 and VCAM-1 in both genotypes. After EAE, immunized WT mice had a sharp increase in the expression of ICAM- 1 and VCAM-1, which were highly colocalized with Cav-1 at the site of infiltration within the lesion, whereas Cav-1 deficiency abolished such changes (Fig. $4 B, C$ ). In line with this, the expression of Cav-1 in the CNS-derived microvessels was upregulated after EAE immunization (Fig. 4D). These results suggest that Cav-1 might modulate adhesion molecules ICAM- 1 and VCAM-1 on endothelial cells and participate in lymphocyte diapedesis. Based on in vivo studies, we used an in vitro coculture system to test the potential function of endothelial Cav-1 in lymphocyte transendothelial migration. Knockdown of Cav-1 expression did not disrupt the cellular membrane integrity (Fig. $4 F$ ) but partially impaired the upregulation of ICAM-1 (Fig. $4 E$ ) in the primed endothelial cell and subsequently attenuated the transendothelial migration of $\mathrm{T}_{\mathrm{H}} 1$ and $\mathrm{T}_{\mathrm{H}} 17$ cells in vitro (Fig. $4 G, H)$. These results, together, suggest the involvement of endothelial Cav-1 in lymphocyte transendothelial migration, probably via regulating adhesion molecules ICAM-1 and VCAM-1.

\section{Discussion}

Increased Cav- 1 expression was previously reported in the spinal cords of EAE lesions (Shin et al., 2005), but its functions in EAE development remain unknown. In the present study, we directly demonstrated the critical roles of Cav-1 in EAE pathogenesis. The expressions of Cav- 1 in serum and spinal cord tissues dramatically increased and reached their maximum coincident with EAE severity. Consistently, Cav-1 KO mice had a remarkable resistance to EAE with lower disease incidence and alleviated clinical symptoms. These results highlight the critical involvement of Cav-1 in EAE and MS pathogenesis.

Cav-1 appears to be a major factor in BBB permeability and lymphocyte transcellular migration, contributing to inflammation and CNS damage. Increased expression of Cav-1 in the neurovascular unit preceded the early BBB breakdown and leukocyte extravasations (Nag et al., 2009; Badaut et al., 2015). In this study, we found that Cav-1 deficiency did not compromise the immune cells priming in the periphery but limited their trafficking. Among various immune cells, antigen-specific CD4 ${ }^{+} \mathrm{T}$ cells, specifically $\mathrm{T}_{\mathrm{H}} 1$ and $\mathrm{T}_{\mathrm{H}} 17$ cells, had been demonstrated as key drivers in EAE and MS development (Huppert et al., 2010). The efficient trafficking of these highly encephalitogenic $T_{H} 1$ and $T_{H} 17$ effector $T$ cells into the $C N S$ is a key prerequisite for CNS demyelination and subsequent paresis or paralysis in MS and EAE (Stromnes et al., 2008; Goverman, 2009). Studies suggested that Cav-1 could positively modulate leukocyte mobilities as the blockage of Cav-1 reduced lymphocyte diapedesis (Zhong et al., 2008; Marmon et al., 2009), although its recovery attenuated such effects (Xu et al., 2013). Consistently, we have demonstrated the positive regulation of Cav-1 and CNS-directed lymphocyte transcellular migration in EAE. Our results showed that Cav-1 deficiency significantly impaired the pathological $\mathrm{T}$ cells trafficking into the CNS, which highlighted the essential involvement of Cav-1 in participating in lymphocyte transmigration pathologically.

A likely mechanism that might explain how Cav-1 behaved during T-cell trafficking pathologically was proposed. ICAM-1 and VCAM-1 are two major molecules essential for efficient lymphocyte diapedesis during inflammation (Greenwood et al., 2011). Millán et al. (2006) and others (Heemskerk et al., 2016; Sun et al., 2016) suggested that the translocation of ICAM-1 to membrane caveolae/Cav-1-enriched domains on endothelial cell is a prerequisite for leukocyte firm adhesion and diapedesis, whereas the abolishment of Cav-1 could diminish the expression of adhesion molecules (Shin et al., 2006; Han et al., 2010) and reduced leukocyte traffickings. In our study, we found highly colocalized Cav-1 with ICAM-1 and VCAM-1 within CNS lesions at the site of leukocyte infiltration pathologically, implicating potential regulatory roles of Cav-1 in these processes. In addition, we also demonstrated that loss of endothelial Cav-1 could downregulate the expression of ICAM- 1 and VCAM- 1 and impaired the efficient trafficking of $\mathrm{T}_{\mathrm{H}} 1$ and $\mathrm{T}_{\mathrm{H}} 17$ cells, both in vivo and in vitro. These results strongly implicate the critical role of Cav-1 in encephalitogenic lymphocyte trafficking into CNS by targeting ICAM- 1 and VCAM-1.

Interestingly, as a cellular trafficking protein, Cav-1 could dissociate and translocate from basal membrane and release into systemic circulation upon stimulations (Liu et al., 2002; Tahir et al., 2008). In the present study, we found that the expression of Cav-1 in serum was simultaneously increased, associated with disease development. This could partially result from the provoked inflammatory responses systemically with some degree of disruptions of membrane caveolae structures, leading to the release of Cav-1 and its appearance in serum. These results imply that the secreted Cav-1 levels in serum could be a potential early biomarker for indexing the clinical severity of EAE and MS. To this respect, further studies are needed to evaluate the clinical relevance of secreted Cav-1 levels in MS patients.

In conclusion, we demonstrate that Cav-1 deficiency could lead to EAE resistance via limiting encephalitogenic T cells trafficking into the CNS by modulating adhesion molecules ICAM-1 and VCAM-1. This study highlights a critical role of Cav-1 in CNS-directed lymphocyte diapedesis and may implicate it as a potential molecular and therapeutic target in neuroinflammatory diseases.

\section{References}

Badaut J, Ajao DO, Sorensen DW, Fukuda AM, Pellerin L (2015) Caveolin expression changes in the neurovascular unit after juvenile traumatic brain injury: signs of barrier healing? Neuroscience 285:215-226. CrossRef Medline

Bullard DC, Hu X, Schoeb TR, Collins RG, Beaudet AL, Barnum SR (2007) Intercellular adhesion molecule-1 expression is required on multiple cell types for the development of experimental autoimmune encephalomyelitis. J Immunol 178:851-857. CrossRef Medline

Engel D, Beckers L, Wijnands E, Seijkens T, Lievens D, Drechsler M, Gerdes N, Soehnlein O, Daemen MJ, Stan RV, Biessen EA, Lutgens E (2011) Caveolin-1 deficiency decreases atherosclerosis by hampering leukocyte influx into the arterial wall and generating a regulatory $\mathrm{T}$-cell response. FASEB J 25:3838-3848. CrossRef Medline

Engelhardt B, Ransohoff RM (2012) Capture, crawl, cross: the T cell code to breach the blood-brain barriers. Trends Immunol 33:579-589. CrossRef Medline

Gaudreault SB, Dea D, Poirier J (2004) Increased caveolin-1 expression in Alzheimer's disease brain. Neurobiol Aging 25:753-759. CrossRef Medline

Ge S, Pachter JS (2004) Caveolin-1 knockdown by small interfering RNA suppresses responses to the chemokine monocyte chemoattractant protein-1 by human astrocytes. J Biol Chem 279:6688-6695. CrossRef Medline

Goverman J (2009) Autoimmune T cell responses in the central nervous system. Nat Rev Immunol 9:393-407. CrossRef Medline

Greenwood J, Heasman SJ, Alvarez JI, Prat A, Lyck R, Engelhardt B (2011) Leucocyte-endothelial cell crosstalk at the blood-brain barrier: a prerequisite for successful immune cell entry to the brain. Neuropathol Appl Neurobiol 37:24-39. CrossRef Medline

Gu Y, Zheng G, Xu M, Li Y, Chen X, Zhu W, Tong Y, Chung SK, Liu KJ, Shen J (2012) Caveolin-1 regulates nitric oxide-mediated matrix metalloproteinases activity and blood-brain barrier permeability in focal cerebral ischemia and reperfusion injury. J Neurochem 120:147-156. CrossRef Medline

Han SG, Eum SY, Toborek M, Smart E, Hennig B (2010) Polychlorinated biphenyl-induced VCAM-1 expression is attenuated in aortic endothelial 
cells isolated from caveolin-1 deficient mice. Toxicol Appl Pharmacol 246:74-82. CrossRef Medline

Heemskerk N, Asimuddin M, Oort C, van Rijssel J, van Buul JD (2016) Annexin A2 limits neutrophil transendothelial migration by organizing the spatial distribution of ICAM-1. J Immunol 196:2767-2778. CrossRef Medline

Huppert J, Closhen D, Croxford A, White R, Kulig P, Pietrowski E, Bechmann I, Becher B, Luhmann HJ, Waisman A, Kuhlmann CR (2010) Cellular mechanisms of IL-17-induced blood-brain barrier disruption. FASEB J 24:1023-1034. CrossRef Medline

Kawakami N, Bartholomäus I, Pesic M, Mues M (2012) An autoimmunity odyssey: how autoreactive T cells infiltrate into the CNS. Immunol Rev 248:140-155. CrossRef Medline

Kim H, Ahn M, Lee J, Moon C, Matsumoto Y, Koh CS, Shin T (2006) Increased phosphorylation of caveolin-1 in the spinal cord of Lewis rats with experimental autoimmune encephalomyelitis. Neurosci Lett 402:76-80. CrossRef Medline

Liu P, Rudick M, Anderson RG (2002) Multiple functions of caveolin-1. J Biol Chem 277:41295-41298. CrossRef Medline

Marmon S, Hinchey J, Oh P, Cammer M, de Almeida CJ, Gunther L, Raine CS, Lisanti MP (2009) Caveolin-1 expression determines the route of neutrophil extravasation through skin microvasculature. Am J Pathol 174:684-692. CrossRef Medline

Millán J, Hewlett L, Glyn M, Toomre D, Clark P, Ridley AJ (2006) Lymphocyte transcellular migration occurs through recruitment of endothelial ICAM-1 to caveola- and F-actin-rich domains. Nat Cell Biol 8:113-123. CrossRef Medline

Nag S, Manias JL, Stewart DJ (2009) Expression of endothelial phosphorylated caveolin-1 is increased in brain injury. Neuropathol Appl Neurobiol 35:417-426. CrossRef Medline

Shin J, Kim J, Ryu B, Chi SG, Park H (2006) Caveolin-1 is associated with VCAM-1 dependent adhesion of gastric cancer cells to endothelial cells. Cell Physiol Biochem 17:10. CrossRef Medline

Shin T, Kim H, Jin JK, Moon C, Ahn M, Tanuma N, Matsumoto Y (2005)
Expression of caveolin-1, -2, and -3 in the spinal cords of Lewis rats with experimental autoimmune encephalomyelitis. J Neuroimmunol 165: 11-20. CrossRef Medline

Steiner O, Coisne C, Cecchelli R, Boscacci R, Deutsch U, Engelhardt B, Lyck R (2010) Differential roles for endothelial ICAM-1, ICAM-2, and VCAM-1 in shear-resistant $\mathrm{T}$ cell arrest, polarization, and directed crawling on blood-brain barrier endothelium. J Immunol 185:4846-4855. CrossRef Medline

Stromnes IM, Cerretti LM, Liggitt D, Harris RA, Goverman JM (2008) Differential regulation of central nervous system autoimmunity by TH1 and TH17 cells. Nat Med 14:337-342. CrossRef Medline

Sun X, Fu Y, Gu M, Zhang L, Li D, Li H, Chien S, Shyy JY, Zhu Y (2016) Activation of integrin alpha 5 mediated by flow requires its translocation to membrane lipid rafts in vascular endothelial cells. Proc Natl Acad Sci U S A 113:769-774. CrossRef Medline

Tahir SA, Yang G, Goltsov AA, Watanabe M, Tabata K, Addai J, Fattah el MA, Kadmon D, Thompson TC (2008) Tumor cell-secreted caveolin-1 has proangiogenic activities in prostate cancer. Cancer Res 68:731-739. CrossRef Medline

Tian XF, Xia XB, Xu HZ, Xiong SQ, Jiang J (2012) Caveolin-1 expression regulates blood-retinal barrier permeability and retinal neovascularization in oxygen-induced retinopathy. Clin Experiment Ophthalmol 40: e58-e66. CrossRef Medline

Xu S, Zhou X, Yuan D, Xu Y, He P (2013) Caveolin-1 scaffolding domain promotes leukocyte adhesion by reduced basal endothelial nitric oxidemediated ICAM-1 phosphorylation in rat mesenteric venules. Am J Physiol Heart Circ Physiol 305:H1484-H1493. CrossRef Medline

Zamvil SS, Steinman L (1990) The T lymphocyte in experimental allergic encephalomyelitis. Annu Rev Immunol 8:579-621. CrossRef Medline

Zhong Y, Smart EJ, Weksler B, Couraud PO, Hennig B, Toborek M (2008) Caveolin-1 regulates human immunodeficiency virus-1 Tat-induced alterations of tight junction protein expression via modulation of the Ras signaling. J Neurosci 28:7788-7796. CrossRef Medline 